\title{
Clinical characteristics and risk factors of COVID-19 patients with chronic hepatitis B: a multi-center retrospective cohort study
}

Jing Wang ${ }^{1, *}$, Zequn $\mathrm{Lu}^{2, *}$, Meng $\mathrm{Jin}^{3, *}$, Ying Wang ${ }^{4, *}$, Kunming Tian ${ }^{5,6, *}, \mathrm{Jun}_{\mathrm{Xiao}}{ }^{1, *}$, Yimin $\mathrm{Cai}^{2, *}$, Yanan Wang ${ }^{1, *}$, Xu Zhang ${ }^{7}$, Tao Chen ${ }^{8}$, Zhi Yao ${ }^{9}$, Chunguang Yang ${ }^{1}$, Renli Deng ${ }^{10}$, Qiang Zhong ${ }^{11}$, Xiongbo Deng ${ }^{12}$, Xin Chen $^{13}$, Xiang-ping Yang ${ }^{14}$, Gonghong Wei ${ }^{15}$, Zhihua Wang $(\bowtie)^{1, \#}$, Jianbo Tian $(\bowtie)^{2, \#}$, Xiao-ping Chen $(\bowtie)^{16, \#}$

${ }^{I}$ Department of Urology, Tongji Hospital, Tongji Medical College, Huazhong University of Science and Technology, Wuhan 430030, China; ${ }^{2}$ Department of Epidemiology and Biostatistics, Key Laboratory for Environment and Health, School of Public Health, Tongji Medical College, Huazhong University of Science and Technology, Wuhan 430030, China; ${ }^{3}$ Department of Respiratory and Critical Care Medicine, Renmin Hospital of Wuhan University, Wuhan 430060, China; ${ }^{4}$ Department of Virology, Wuhan Centers for Disease Prevention and Control, Wuhan 430024, China; ${ }^{5}$ Institute of Reproductive Health, Tongji Medical College, Huazhong University of Science and Technology, Wuhan 430030, China; ${ }^{6}$ School of Nursing, Zunyi Medical University, Zunyi 563099, China; ${ }^{7}$ Key Laboratory of Environment and Health, Ministry of Education \& Ministry of Environmental Protection, and State Key Laboratory of Environmental Health (Incubating), School of Public Health, Tongji Medical College, Huazhong University of Science and Technology, Wuhan 430030, China; ${ }^{8}$ Department and Institute of Infectious Disease, Tongji Hospital, Tongji Medical College, Huazhong University of Science and Technology, Wuhan 430030, China; ${ }^{9}$ Department of Respirology and Tuberculosis Specialty, Wuhan Pulmonary Hospital, Wuhan 430030, China; ${ }^{10}$ The Fifth Affiliated (Zhuhai) Hospital of Zunyi Medical University, Zhuhai 519199, China; ${ }^{11}$ Department of Critical Care Medicine, Tongji Hospital, Tongji Medical College, Huazhong University of Science and Technology, Wuhan 430030, China; ${ }^{12}$ Department of Radiology, Wuhan Jinyintan Hospital, Wuhan 430048, China; ${ }^{13}$ Department of Oncology, Tongji Hospital, Tongji Medical College, Huazhong University of Science and Technology, Wuhan 430030, China; ${ }^{14}$ Department of Immunology, Tongji Medical College, Huazhong University of Science and Technology, Wuhan 430030, China; ${ }^{15}$ Biocenter Oulu, Faculty of Biochemistry and Molecular Medicine, University of Oulu, Oulu 90014, Finland; ${ }^{16}$ Hepatic Surgery Center, Tongji Hospital, Tongji Medical College, Huazhong University of Science and Technology, Wuhan 430030, China

(C) Higher Education Press 2021

\begin{abstract}
The coronavirus disease 2019 (COVID-19) has spread globally. Although mixed liver impairment has been reported in COVID-19 patients, the association of liver injury caused by specific subtype especially chronic hepatitis B (CHB) with COVID-19 has not been elucidated. In this multi-center, retrospective, and observational cohort study, $109 \mathrm{CHB}$ and 327 non-CHB patients with COVID-19 were propensity score matched at an approximate ratio of 3:1 on the basis of age, sex, and comorbidities. Demographic characteristics, laboratory examinations, disease severity, and clinical outcomes were compared. Furthermore, univariable and multivariable logistic and Cox regression models were used to explore the risk factors for disease severity and mortality, respectively. A higher proportion of CHB patients ( 30 of $109(27.52 \%)$ ) developed into severe status than non-CHB patients $(17$ of $327(5.20 \%))$. In addition to previously reported liver impairment markers, such as alanine aminotransferase, aspartate aminotransferase, alkaline phosphatase, and total bilirubin, we identified several novel risk factors including elevated lactate dehydrogenase $(\geqslant 245 \mathrm{U} / \mathrm{L}$, hazard ratio $(\mathrm{HR})=8.639,95 \%$ confidence interval $(C I)=2.528-29.523 ; P<0.001)$ and coagulation-related biomarker $D$-dimer $(\geqslant 0.5 \mu \mathrm{g} / \mathrm{mL}, \mathrm{HR}=4.321$, 95\% $\mathrm{CI}=1.443-12.939 ; P=0.009)$ and decreased albumin $(<35 \mathrm{~g} / \mathrm{L}, \mathrm{HR}=\mathbf{0 . 1 3 1}, 95 \% \mathrm{CI}=\mathbf{0 . 0 4 8}-0.361 ; P$ $<0.001)$ and albumin/globulin ratio $(<1.5, \mathrm{HR}=0.123,95 \% \mathrm{CI}=0.017-0.918 ; P=0.041)$. In conclusion, COVID19 patients with CHB were more likely to develop into severe illness and die. The risk factors that we identified may be helpful for early clinical surveillance of critical progression.
\end{abstract}

Received November 22, 2020; accepted March 15, 2021

Correspondence: Zhihua Wang, zhwang_hust@hotmail.com;

Jianbo Tian, tianjb@hust.edu.cn;

Xiao-ping Chen, chenxpchenxp@163.com

${ }^{*}$ These authors contributed equally as co-first authors.

${ }^{\#}$ These authors contributed equally as co-corresponding authors. 
Keywords COVID-19; chronic hepatitis B; liver injury; coagulation dysfunction

\section{Introduction}

The coronavirus disease 2019 (COVID-19) caused by severe acute respiratory syndrome coronavirus 2 (SARSCoV-2) has become a global threat to human health. As of November 19, 2020, the number of confirmed cases reached 55928 327, and the COVID-19 pandemic caused the death of 1344003 patients worldwide [1]. To control the increase of cases, the vulnerable population including the elderly and people with comorbidities should be well protected from SARS-CoV-2 infection.

Recent studies about the clinical characteristics of COVID-19 patients have shown that diabetes, hypertension, and tumors are common comorbidities among them, and patients with these pre-existing conditions have a higher risk of developing severe events and death [2-4]. As a major disease burden globally, chronic liver disease, including chronic viral hepatitis, non-alcoholic fatty liver disease, and alcohol-related liver disease, has affected approximately 1.5 billion people worldwide in 2017 [5]. Chronic hepatitis B (CHB) is an important type of liver injury with about 391 million people infected globally at risk of developing decompensated liver disease and hepatocellular carcinoma [5]. Accumulating evidence has shown that liver impairment frequently occurs in COVID19 patients [6,7]. Additionally, a pathological study of liver biopsy specimens from COVID-19 patients showed moderate microvesicular steatosis and mild lobular and portal activity, indicating that SARS-CoV-2 may lead to acute liver damage [8]. However, most of the reports focused on mixed liver injury rather than specific subtype that possesses distinct pathogenesis. The possible association between CHB and COVID-19 remains to be fully elucidated. A detailed description of the clinical characteristics, laboratory parameters (e.g., inflammatory cytokines, immune cells, and liver injury biomarkers), and clinical interventions of COVID-19 patients with CHB is urgently needed, especially for the risk factors correlated with disease severity and clinical outcomes, to protect $\mathrm{CHB}$ patients from SARS-CoV-2 infection, monitor disease progression, and make treatment protocols for this particular population.

In this study, we carried out a multi-center, retrospective, and observational study from three hospitals that were designated to treat COVID-19 patients in Wuhan, China. We aimed to systematically characterize the clinical features and identify risk factors for disease severity and mortality of COVID-19 patients with CHB.

\section{Materials and methods}

\section{Study design and participants}

This multi-center, retrospective, and observational study was conducted in three hospitals (Wuhan, China) that were designated to treat COVID-19 patients: Tongji Hospital, Wuhan Jinyintan Hospital, and Wuhan Pulmonary Hospital. These hospitals are located across Wuhan and admitted 7013 patients confirmed with SARS-CoV-2 infection from January 13 to April 15, 2020. Among these patients, all patients with CHB $(N=109)$ were enrolled, and a total of 327 patients without $\mathrm{CHB}$ were statistically matched by propensity score matching [9] at an approximate ratio of $3: 1$ on the basis of age, sex, and comorbidities, including hypertension, diabetes, coronary heart disease, cerebral infarction, and pulmonary tuberculosis, which have been reported to be risk factors for severity or death of COVID$19[3,4]$. In addition, patients with other pre-existing liver diseases, such as liver cirrhosis, fatty liver disease, liver cancer, and other advanced liver diseases, were excluded. The patients with CHB were grouped into non-severe $(N=$ $79)$ and severe groups $(N=30)$ on admission. This study was approved by the Ethics Committee of Tongji Hospital, Tongji Medical College, Huazhong University of Science and Technology and granted a waiver of informed consent from study participants. A detailed flowchart of the study design is shown in Fig. 1. A list including each site from which patients were recruited, the name of the principal investigator responsible for this site, and the number of patients in each site is presented in Table S1.

\section{Data collection}

The epidemiological, clinical, radiological, laboratory, clinical treatment, and clinical outcome data of all patients with laboratory-confirmed SARS-CoV-2 were obtained with data collection forms from the electronic medical records of hospitals. The admission and in-hospital data of these patients were collected, reviewed, and verified by a trained team of physicians (Qiang Zhong, Tao Chen, and Chunguang Yang). Detailed and standardized information such as demographic data, comorbidities, initial symptoms, vital signs, chest computed tomographic (CT), and past anti-hepatitis therapies was recorded within $24 \mathrm{~h}$ after admission. The complications, treatments, and clinical outcomes were monitored in the hospital. Hepatitis B virus serological markers (HBsAg, $\mathrm{HBsAb}, \mathrm{HBeAg}, \mathrm{HBeAb}$, 


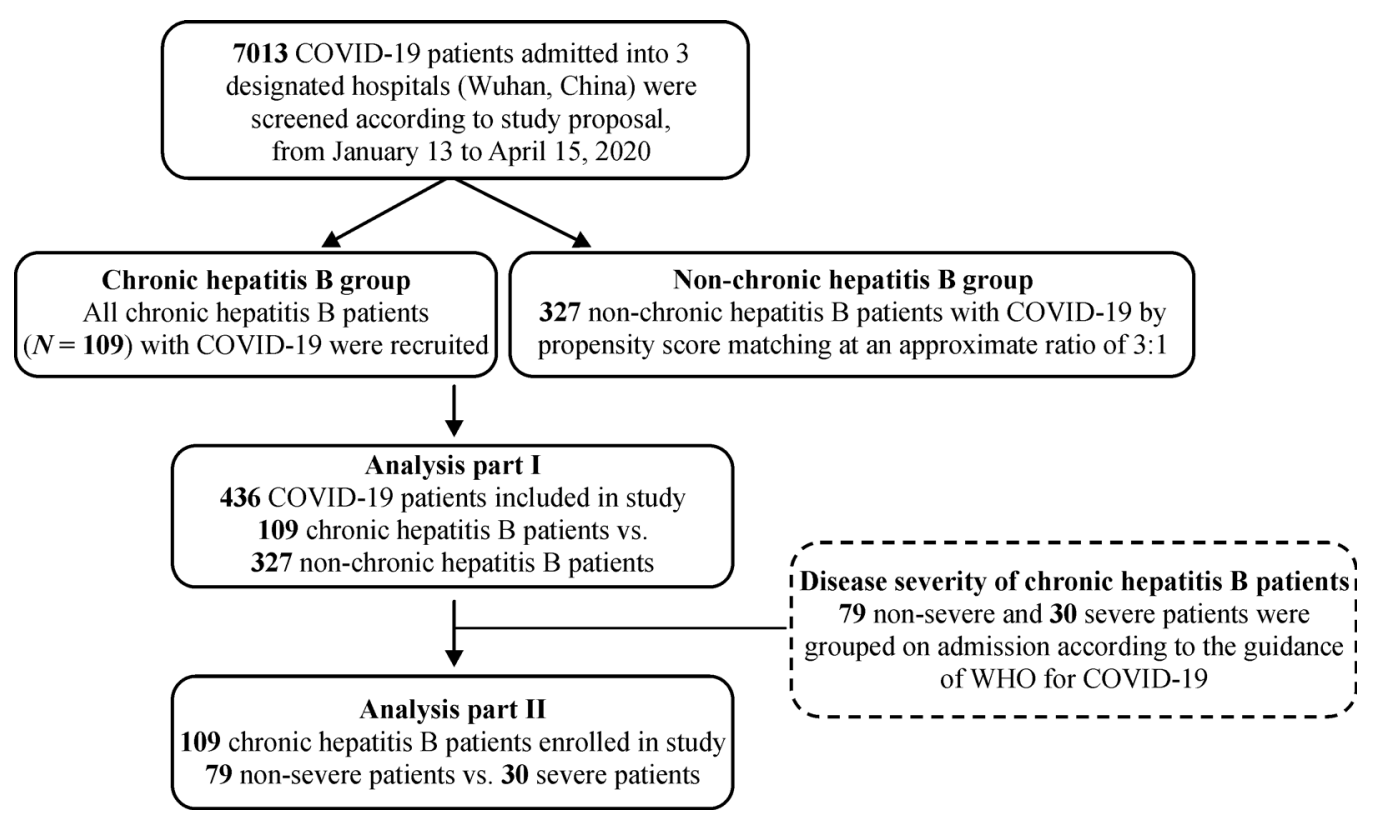

Fig. 1 Flowchart of this study. This multi-center, retrospective, and observational study was conducted at three hospitals designated for COVID-19 treatment. From January 13 to April 15, 2020, 7013 laboratory-confirmed COVID-19 patients were admitted at these hospitals. Among them, all CHB patients $(N=109)$ were ultimately enrolled, and 327 non-CHB patients that were statistically matched by propensity score at an approximate ratio of $3: 1$ on the basis of age, sex, and comorbidities were recruited. To explore the potential risk factors associated with COVID-19 severity in CHB patients, we classified these patients into non-severe $(n=79)$ and severe groups $(n=30)$ at admission according to the guidance of the WHO for COVID-19 and the Seventh Revised Trial Version of the COVID-19 Diagnosis and Treatment Guidance (2020) of China.

$\mathrm{HBcAb}$, and HBV DNA) were collected or recorded within $24 \mathrm{~h}$ after admission. Laboratory examinations including routine blood test, lymphocyte subsets, inflammatory or infection-related biomarkers, and cardiac/renal/ liver/coagulation function tests were recorded on the first diagnosis date within $24 \mathrm{~h}$ after admission.

\section{Definitions}

CHB is defined as a chronic necroinflammatory disease of the liver caused by persistent infection with HBV [10]. In addition, patients with other pre-existing liver diseases, such as liver cirrhosis, fatty liver disease, liver cancer, and other advanced liver diseases, were excluded in our study. Patients aged $\geqslant 18$ years were enrolled. All enrolled patients were classified as having non-severe or severe COVID-19 on admission. We defined the disease severity of COVID-19 on the basis of the WHO guidelines [11] and the Seventh Revised Trial Version of the COVID-19 Diagnosis and Treatment Guidance (2020) of China [12], with the following criteria: respiratory rate $\geqslant 30$ breath/min, oxygen saturation $\leqslant 93 \%$ at a rest state, ratio of arterial partial pressure of oxygen $\left(\mathrm{PaO}_{2}\right)$ and oxygen concentration $\left(\mathrm{FiO}_{2}\right) \leqslant 300 \mathrm{mmHg}$, or patients with $>50 \%$ lesions progressing within 24 to $48 \mathrm{~h}$ in chest CT imaging. The definition of disseminated intravascular coagulation (DIC) was based on the scoring system recommended by the International Society on Thrombosis and Haemostasis (ISTH) Subcommittee of the Scientific and Standardization Committee. DIC was diagnosed by increased levels of platelets, clotting factors, and other blood components. Patients with a score $>5$ were diagnosed as DIC [13]. The time of follow-up was defined as the duration from admission to outcomes of patients. The clinical outcomes were classified into survivors and nonsurvivors, and survivors were defined as patients who were discharged from the hospital or still hospitalized at the end of the study. No cases were lost to follow-up in this study, which was attributed to standardized government management and close tracking of the COVID-19 pandemic.

\section{Laboratory procedures}

The concentrations of interleukin- $1 \beta$ (IL-1 $\beta$ ), IL-2 receptor (IL-2R), IL-6, IL-8, IL-10, and tumor necrosis factor- $\alpha$ (TNF- $\alpha$ ) were detected in serum samples by a fully automated analyzer (Cobas e602; Roche Diagnostics, Indianapolis, IN, USA) on the basis of the chemiluminescence immunoassay method [14]. Kits for IL-1 $\beta$, IL-2R, IL-8, IL-10, and TNF- $\alpha$ were purchased from DiaSorin (Vercelli, Italy), and the IL-6 kit was purchased from Roche Diagnostics. 


\section{Confirmation of COVID-19}

SARS-CoV-2 was determined by real-time reverse transcription PCR [15]. Two pairs of primers targeting the open reading frame $1 \mathrm{ab}(\mathrm{ORF} 1 \mathrm{ab})$ and the nucleocapsid protein $(\mathrm{N})$ were amplified and examined. The corresponding sequences for target 1 (ORF1ab) were 5'-CCCTGTGGGTTTTACACTTAA-3' (F), 5'-ACGATT-GTGCATCAGCTGA-3' (R), and 5'-VIC-CCGTCTGC-GGTATGTGGAAAGGTTATGG-BHQ1-3' (probe), and those for target $2(\mathrm{~N})$ were $5^{\prime}$-GGGGAACTTCTCCTGCTAGAAT3' (F), 5'-CAGACATTTTGCTCTCAAGCTG-3' (R), and 5'-FAM- TTGCTGCTGCTTGACAGATT-TAMRA-3' (probe). These diagnostic criteria were based on the recommendations by the National Centers for Disease Control and Prevention of China. Each sample was duplicated in triplicate with positive and negative control sets, as suggested.

\section{Statistical analysis}

Continuous variables belonging to non-normal distribution were described as the median and interquartile range (IQR). Categorical variables were expressed as number (\%). For continuous variables, the Mann-Whitney U nonparameter test was used for non-normally distributed data. Pearson's $\chi^{2}$ test or Fisher's exact test was applied for categorical variables. To explore the risk factors associated with severity in COVID-19 patients with CHB, univariable and multivariable logistic regression was employed to estimate the odds ratios (ORs) and 95\% confidence intervals (CIs) adjusting for age, sex, and comorbidities. The univariable and multivariable Cox proportional hazards models adjusting for the abovementioned confounders were used to determine the hazard ratios (HRs) and $95 \%$ CIs for the death risk of COVID-19 patients. We conducted a power analysis for the severity or death of COVID-19 in CHB and non-CHB patients using power calculations for the chi-square test method, and the results were 0.950 and 0.982 , respectively. A two-sided $P$ value $<0.05$ was considered statistically significant. All statistical analyses were performed using SPSS (23.00) and $\mathrm{R}$ (3.50).

\section{Results}

\section{Clinical characteristics of COVID-19 patients with CHB}

A total of 109 (109 of $436(25.00 \%)$ ) CHB patients and 327 (327 of $436(75.00 \%)$ ) non-CHB patients that were statistically matched on the basis of age, sex, and comorbidities were enrolled. The CHB patients with COVID-19 were further classified into non-severe (79 of $109(72.48 \%)$ ) and severe (30 of $109(27.52 \%)$ ) groups.

As shown in Table S2, compared with COVID-19 patients without $\mathrm{CHB}$, CHB patients had a higher prevalence of dyspnea (60 of $109(55.05 \%)$ vs. 141 of 327 (43.12\%); $P=0.031$ ). Moreover, abnormalities of chest $\mathrm{CT}$ images such as ground-glass opacity and patchy shadows were almost seen in all COVID-19 patients; however, the differences were not significant between both groups. Notably, a manifestation of dyspnea symptoms, ground-glass opacity in chest radiograph, and lower oxygen saturation were more pronounced in severe cases compared with non-severe CHB patients (Table S3).

We observed substantial differences in laboratory findings between non-CHB and CHB patients, especially in liver impairment-related indicators. The levels of alanine aminotransferase (ALT, 31.00 vs. $18.00 \mathrm{U} / \mathrm{L} ; P<0.001$ ), aspartate aminotransferase (AST, 35.50 vs. $21.00 \mathrm{U} / \mathrm{L}$; $P<0.001$ ), total bilirubin (TBIL, 10.25 vs. $8.20 \mu \mathrm{mol} / \mathrm{L}$; $P<0.001$ ), direct bilirubin (DBIL, 3.60 vs. $3.30 \mu \mathrm{mol} / \mathrm{L}$; $P=0.003)$, total bile acid ( 4.70 vs. $3.30 \mu \mathrm{mol} / \mathrm{L} ; P=0.006$, Table S2), lactate dehydrogenase (LDH, 238.00 vs. 211.00 $\mathrm{U} / \mathrm{L} ; P=0.019$ ), and alkaline phosphatase (ALP, 68.00 vs. $62.50 \mathrm{U} / \mathrm{L} ; P=0.049$ ) were substantially elevated in CHB patients. Conversely, biomarkers reflecting synthetic function, such as albumin (ALB, 33.95 vs. $39.40 \mathrm{~g} / \mathrm{L}$; $P<0.001$ ), total protein (TP, 61.55 vs. $69.30 \mathrm{~g} / \mathrm{L}$; $P<0.001$ ), and the ratio of albumin and globulin (ALB/ GLO, 1.23 vs. $1.35 ; P<0.001$ ) were decreased in CHB patients compared with non-CHB patients. Remarkably, the coagulation-related biomarker D-dimer $(0.95$ vs. 0.36 $\mu \mathrm{g} / \mathrm{mL} ; P<0.001$ ) and prothrombin time (PT, 14.40 vs. $13.50 \mathrm{~s} ; P<0.001)$ were higher in CHB patients than in non-CHB patients. Additionally, CHB patients had an increased level of creatine kinase-MB (CK-MB, 1.10 vs. $0.70 \mathrm{U} / \mathrm{L} ; P<0.001$ ) than non-CHB patients (Table 1 ).

We also observed that the serum levels of inflammatory cytokines including IL-6 (9.92 vs. $3.08 \mathrm{pg} / \mathrm{mL} ; P<0.001)$ and TNF- $\alpha(9.15$ vs. $7.00 \mathrm{pg} / \mathrm{mL} ; P<0.001)$ were significantly elevated in $\mathrm{CHB}$ patients compared with non-CHB patients. Moreover, CHB patients had higher levels of high-sensitivity C-reactive protein (hs-CRP, 19.25 vs. $3.70 \mathrm{mg} / \mathrm{L} ; P<0.001)$, an infection-related biomarker, and neutrophil $\left(4.26\right.$ vs. $3.25 \times 10^{9} / \mathrm{L}$; $P<0.001)$ and leukocyte counts $\left(6.15\right.$ vs. $5.24 \times 10^{9} / \mathrm{L}$; $P<0.001)$. Conversely, in terms of immune cell counts, the baseline counts of lymphocytes $\left(1.24\right.$ vs. $1.42 \times 10^{9} / \mathrm{L}$; $P<0.001), \mathrm{CD}^{+}{ }^{+} \mathrm{CD} 19^{-} \mathrm{T}$ cells $(552.80$ vs. $1112.00 / \mu \mathrm{L}$, $P<0.001)$, and $\mathrm{CD}^{+} \mathrm{T}$ cells $(481.00$ vs. $564.00 / \mu \mathrm{L}$, $P=0.001)$ were drastically decreased in CHB patients (Table 1). Notably, the aggravated inflammatory responses and multi-organ damages, especially liver injury and coagulation dysfunction, were more pronounced in severe 


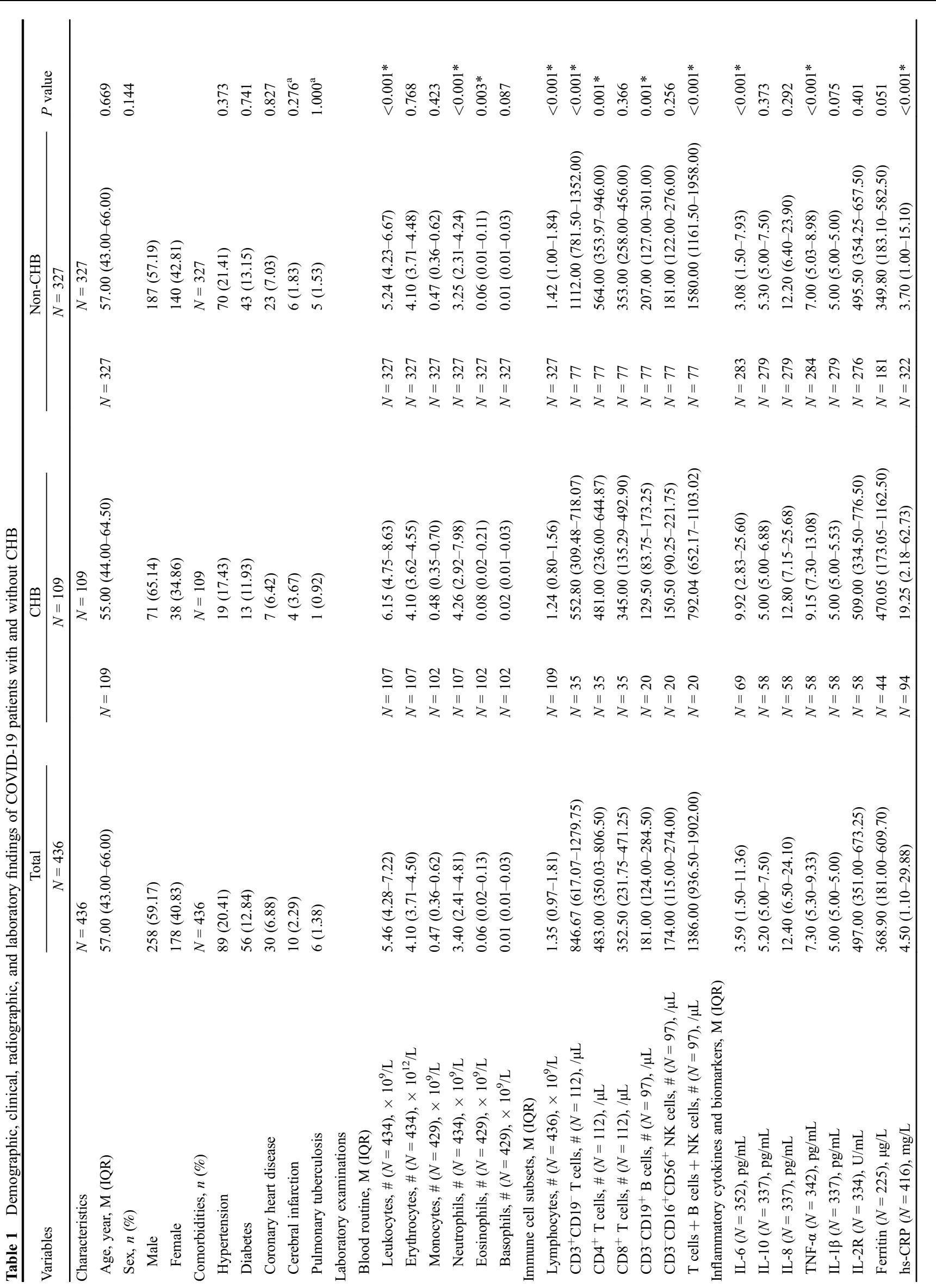




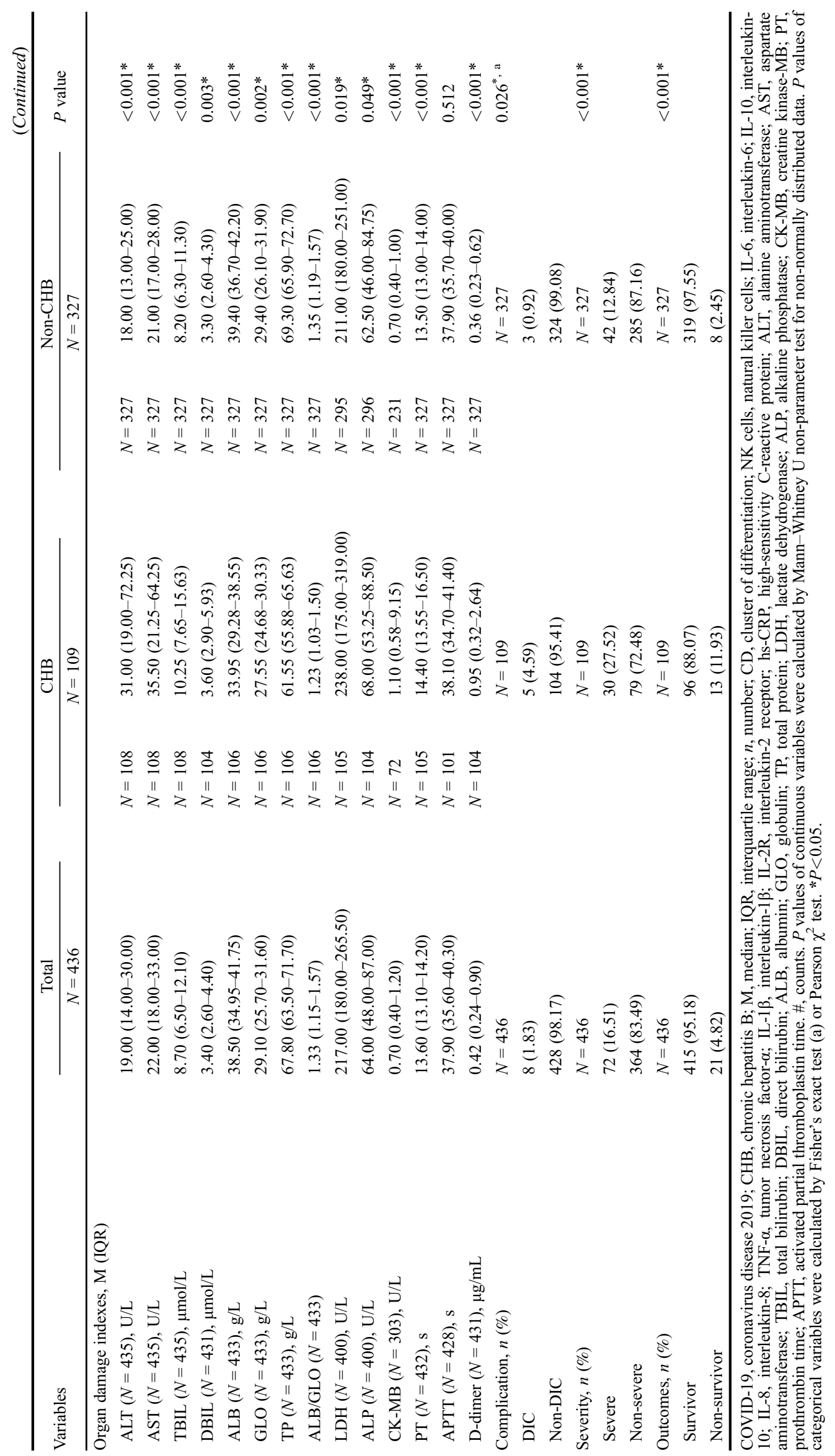


COVID-19 patients with CHB compared with non-severe CHB patients (Table 2). Other laboratory indices or organ damage biomarkers in $\mathrm{CHB}$ and non-CHB patients and severe and non-severe $\mathrm{CHB}$ patients are presented in Tables S2 and S3.

Importantly, we found that CHB patients (30 of 109 $(27.52 \%))$ had a higher proportion of developing into severe status than non-CHB patients (42 of 327 (12.84\%)), and the odds of progressing into severe illness was 2.632 times higher $(95 \% \mathrm{CI}=1.526-4.539 ; P<0.001)$. The medians (IQRs) of follow-up were 19.00 (13.00-29.00) days and 17.00 (9.00-22.00) days in CHB and non-CHB patients with COVID-19, respectively $(P=0.007)$. Compared with non-CHB patients, CHB patients (13 of $109(11.93 \%)$ vs. 8 of $327(2.45 \%))$ had higher mortality (Table 1). As expected, severe patients had higher observed death rates (7 of $30(23.33 \%)$ vs. 6 of 79 (7.59\%); $P=0.042$ ) than non-severe patients with CHB (Table 2). In terms of hepatitis B virus serological markers, compared with non-severe patients, severe patients had a higher proportion of HBeAg-positive patients (11 of 30 $(36.67 \%)$ vs. $(0 \%) ; P<0.001)$ and lower proportion of HBeAb-positive patients (18 of $30(60.00 \%)$ vs.74 of 79 (93.67\%); $P<0.001$, Table 2).

\section{Complication and clinical treatments of COVID-19 patients with $\mathrm{CHB}$}

SARS-CoV-2 infection can cause pulmonary and multisystem inflammation, leading to critical complications, as summarized in Tables 1 and 2. Consistent with the findings of organ damage biomarkers, the complication of DIC (5 of $109(4.59 \%)$ vs. 3 of $327(0.92 \%) ; P=0.026)$ frequently occurred in CHB patients than in non-CHB patients with COVID-19.

In terms of anti-hepatitis therapies for COVID-19 patients with CHB (Table 2), severe patients received more past anti-HBV therapy prior to COVID-19 (22 of 30 $(73.33 \%)$ vs. 52 of $79(65.82 \%))$ and liver protective therapy (27 of $30(90.00 \%)$ vs. 59 of $79(74.68 \%))$. In terms of clinical treatments for COVID-19 patients, almost half of the COVID-19 patients received antibiotic and antiviral treatment, and the differences of the use of these therapies were not significant in $\mathrm{CHB}$ and non-CHB groups; however, intravenous immunoglobulin therapy (51 of $109(46.79 \%)$ vs. 78 of 327 (23.85\%); $P<0.001$ ) was more frequently used in CHB patients than in non-CHB patients (Table 3). The same tendency could be found in the comparison of severe and non-severe COVID-19 patients with CHB. Ventilation treatment was the conventional non-drug therapy. A higher proportion of COVID-19 patients with CHB received mechanical ventilation (25 of $109(22.49 \%)$ vs. 26 of $327(7.95 \%) ; P<0.001)$ than COVID-19 patients without CHB.

\section{Risk factors associated with the severity and outcome of COVID-19 patients with CHB}

Furthermore, multivariate logistic models were applied to explore the risk factors associated with disease severity of COVID-19 in CHB patients with adjustment of age, sex, and comorbidities. As shown in Table 4, we found that patients with elevated ALT $(\mathrm{OR}=1.012,95 \% \mathrm{CI}=1.004$ $1.020 ; P=0.003)$, AST $(\mathrm{OR}=1.007,95 \% \mathrm{CI}=1.001-$ $1.013 ; P=0.027)$, ALP $(\mathrm{OR}=1.009,95 \% \mathrm{CI}=1.001-$ $1.017 ; P=0.030)$, TBIL $(\mathrm{OR}=1.054,95 \% \mathrm{CI}=1.018$ $1.091 ; P=0.003)$, and $\mathrm{LDH}(\mathrm{OR}=1.004,95 \% \mathrm{CI}=$ $1.001-1.007 ; P=0.021$ ) presented higher risks for disease severity among COVID-19 patients with CHB. Conversely, the levels of ALB $(\mathrm{OR}=0.803,95 \% \mathrm{CI}=0.723-$ $0.891 ; P<0.001)$ and $\mathrm{ALB} / \mathrm{GLO}(\mathrm{OR}=0.062,95 \% \mathrm{CI}=$ $0.010-0.385 ; P=0.003$ ) were significantly associated with lower severity of COVID-19 in CHB patients. Noticeably, $\mathrm{D}$-dimer $(\mathrm{OR}=1.100,95 \% \mathrm{CI}=1.022-1.185 ; P=0.012)$, a coagulation-related biomarker, was significantly elevated in COVID-19 patients with CHB. Additionally, elevated pro-inflammatory cytokines (e.g., TNF- $\alpha$ and IL-6) and infection-related factors (e.g., hs-CRP) and decreased immune cell subsets such as lymphocytes (including $\mathrm{CD}^{+}{ }^{+} \mathrm{CD} 19^{-} \mathrm{T}$ cells and $\mathrm{CD} 4^{+} \mathrm{T}$ cells) were remarkably associated with increased severity of the disease. Elevated levels of hs-cTnI (a cardiac-related indicator) and baseline counts of neutrophils would increase the risk of disease severity. Similarly, the aggravated inflammatory markers decreased immune cells, and abnormal organ damage indices, especially liver and coagulation-related biomarkers, were associated with the risk of COVID-19 death (Table S4).

A higher proportion of CHB patients (13 of 109 $(11.93 \%)$ ) eventually died than non-CHB patients (8 of $327(2.45 \%)$ ), and the odds of dying was 3.748 times higher $(95 \% \mathrm{CI}=1.522-9.234 ; P=0.004$, Fig. 2A). Previous reports indicated that ALT, AST, ALP, and TBIL are associated with the mortality risk of COVID-19 [16], which was also confirmed in our study (Fig. 2B-2E). Apart from these indices, we identified that patients with higher levels of $\mathrm{LDH}(\mathrm{HR}=8.639,95 \% \mathrm{CI}=2.528-29.523$; $P<0.001)$ and $\mathrm{D}$-dimer $(\mathrm{HR}=4.321,95 \% \mathrm{CI}=1.443-$ $12.939 ; P=0.009)$ had higher risks of dying from COVID19 (Fig. $2 \mathrm{~F}$ and $2 \mathrm{G})$, whereas ALB $(\mathrm{HR}=0.131,95 \% \mathrm{CI}=$ $0.048-0.361 ; P<0.001)$ and ALB/GLO $(\mathrm{HR}=0.123$, 95\% CI $=0.017-0.918 ; P=0.041)$ presented a protective effect for prognosis (Fig. 2H and 2I).

\section{Discussion}

Several studies have reported that mixed liver impairment frequently occurs in COVID-19 patients, while the clinical characteristics and risk factors for COVID-19 patients with 


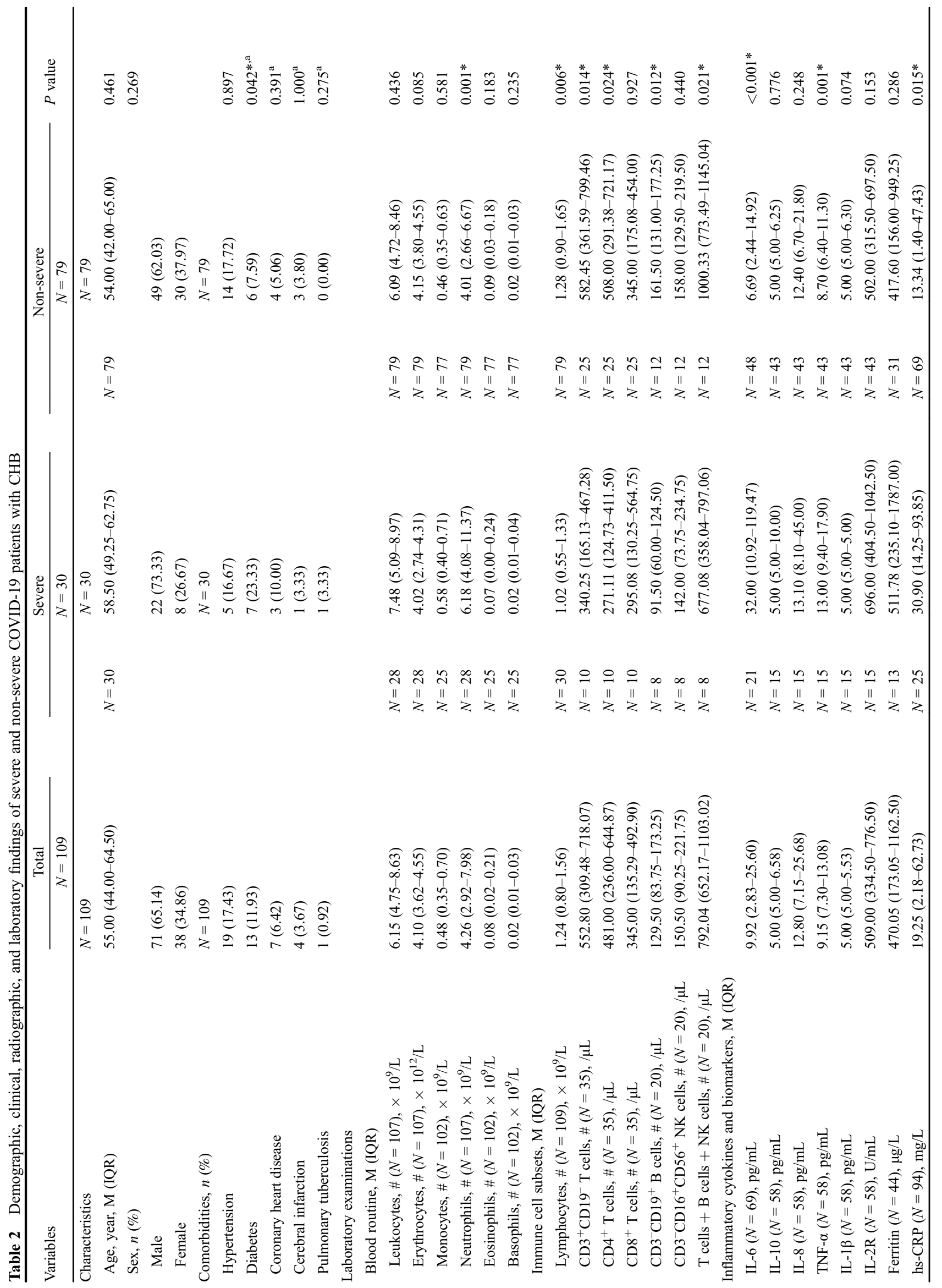




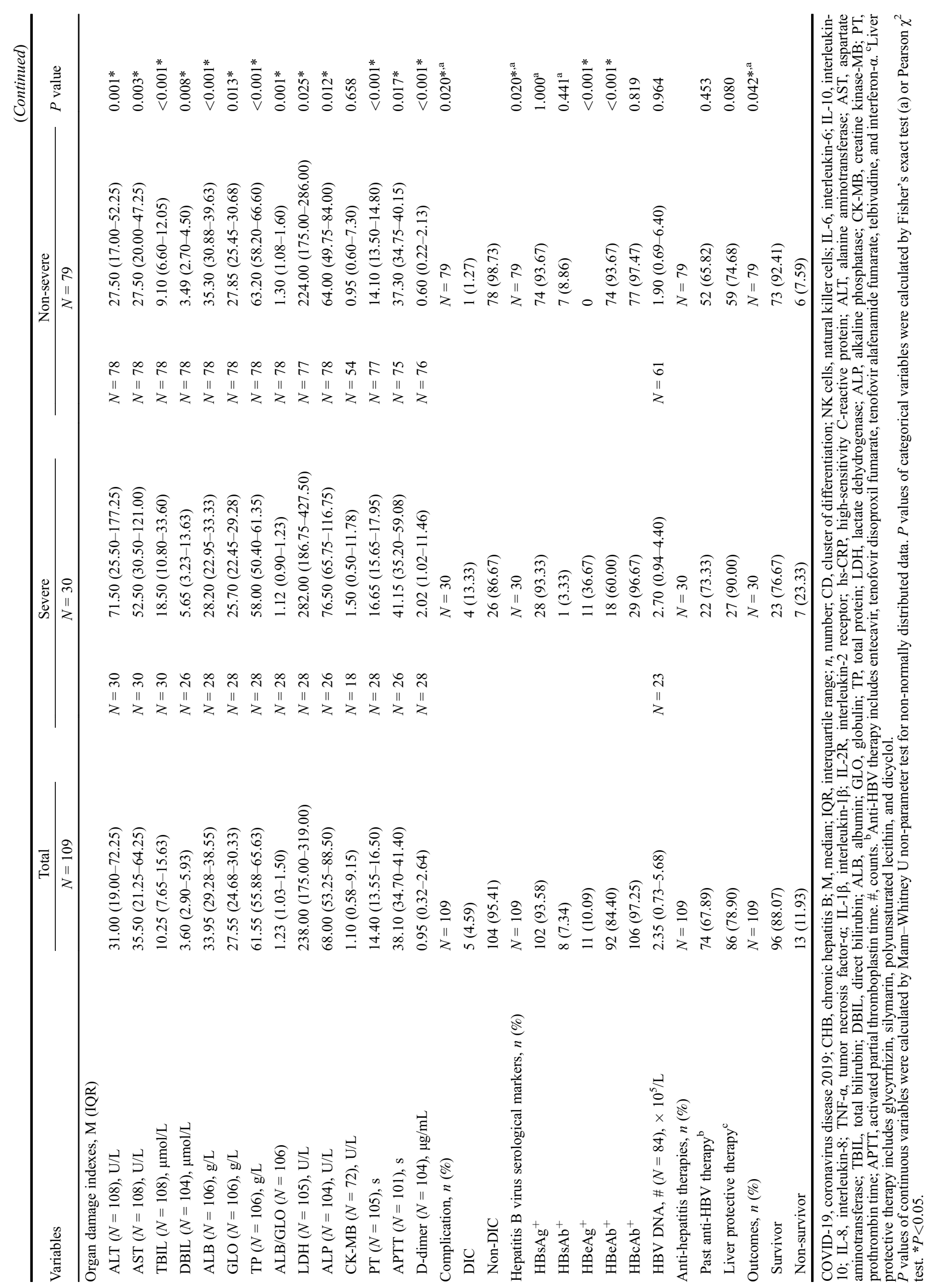


pre-existing conditions of $\mathrm{CHB}$ remain to be fully elucidated. Here, through integrating multi-center clinical data, we found that compared with non-CHB patients, COVID-19 patients with CHB possessed a higher risk of acquiring severe illness or death, which was associated with liver injury related to viral infection, disturbances of coagulation hemostasis, and abnormal inflammatory response. In addition to the reported liver impairment markers, such as ALT, AST, ALP, and TBIL, several novel risk factors including elevated $\mathrm{LDH}$ and $\mathrm{D}$-dimer and decreased ALB and ALB/GLO were shown to be closely correlated with severity or mortality in COVID-19 patients with $\mathrm{CHB}$, which would be of great value in the early surveillance of critical progression and implementation of effective prevention strategies and interventions for this susceptible population.

The enrolled COVID-19 patients with and without CHB were statistically matched to exclude the effects of common confounders, such as age, sex, and other comorbidities. We noted that COVID-19 patients with CHB presented aggravated pro-inflammatory cytokines and multiple organ damage, especially liver injury and coagulation dysfunction, compared with patients without CHB. These findings provided supporting evidence that COVID-19 patients with CHB were more likely to develop into severe illness or die. Notably, all patients in the study were symptomatic, and thus a screen or an epidemiological study in CHB patients is warranted to reflect the rate of asymptomatic infection.

Furthermore, we used multivariable logistic and Cox regression models to explore the factors related to COVID19 severity and death among patients with CHB, respectively, with adjustment of age, sex, and other comorbidities. Liver injury-related factors such as ALT, AST, ALP, and TBIL, which were previously reported to be associated with death risk of COVID-19 patients [16], were also confirmed in the present study. We further identified several novel risk factors including elevated LDH and decreased ALB and ALB/GLO. As a potential novel biomarker found in our study, LDH not only plays a significant role in glucose metabolism through catalyzing pyruvate to lactate, but also regulates the immune response

Table 3 Clinical treatments and complications of COVID-19 patients

\begin{tabular}{|c|c|c|c|c|}
\hline \multirow{3}{*}{ Variables } & \multicolumn{4}{|c|}{ All patients } \\
\hline & Total & $\mathrm{CHB}$ & Non-CHB & \multirow{2}{*}{$P$ value } \\
\hline & $N=436$ & $N=109$ & $N=327$ & \\
\hline \multicolumn{5}{|l|}{ Treatments $^{\mathrm{b}}, n(\%)$} \\
\hline Antiviral therapy & $194(44.50)$ & $49(44.95)$ & $145(44.34)$ & 0.911 \\
\hline Antibiotics & $287(65.83)$ & $74(67.89)$ & $213(65.14)$ & 0.600 \\
\hline Intravenous immunoglobulin therapy & $129(29.59)$ & $51(46.79)$ & $78(23.85)$ & $<0.001 *$ \\
\hline Glucocorticoid therapy & $128(29.36)$ & $42(38.53)$ & $86(26.30)$ & $0.015^{*}$ \\
\hline High-flow oxygen therapy & $19(4.36)$ & $6(5.50)$ & $13(3.98)$ & 0.587 \\
\hline Mechanical ventilation & $51(11.70)$ & $25(22.49)$ & $26(7.95)$ & $<0.001^{*}$ \\
\hline Non-invasive & $26(5.96)$ & $7(6.42)$ & $19(5.81)$ & 0.815 \\
\hline Invasive & $11(2.52)$ & $4(3.67)$ & $7(2.14)$ & $0.479^{\mathrm{a}}$ \\
\hline \multirow[t]{2}{*}{ Transfusion } & $22(5.05)$ & $7(6.42)$ & $15(4.59)$ & 0.448 \\
\hline & \multicolumn{4}{|c|}{ CHB patients } \\
\hline \multirow[t]{2}{*}{ Variables } & Total & Severe & Non-severe & \multirow{2}{*}{$P$ value } \\
\hline & $N=109$ & $N=30$ & $N=79$ & \\
\hline \multicolumn{5}{|l|}{ Treatments $^{\mathrm{b}}, n(\%)$} \\
\hline Antiviral therapy & $49(44.95)$ & $16(53.33)$ & $33(41.77)$ & 0.278 \\
\hline Antibiotics & $74(67.89)$ & $22(73.33)$ & $52(65.82)$ & 0.453 \\
\hline Intravenous immunoglobulin therapy & $51(46.79)$ & $20(66.67)$ & $31(39.24)$ & $0.010^{*, \mathrm{a}}$ \\
\hline Glucocorticoid therapy & $42(38.53)$ & $14(46.67)$ & $28(35.44)$ & 0.282 \\
\hline High-flow oxygen therapy & $6(5.50)$ & $1(3.33)$ & $5(6.33)$ & $1.000^{\mathrm{a}}$ \\
\hline Mechanical ventilation & $25(22.94)$ & $9(30.00)$ & $16(20.25)$ & 0.280 \\
\hline Non-invasive & $7(6.42)$ & $6(20.00)$ & $1(1.27)$ & $0.002^{*, \mathrm{a}}$ \\
\hline Invasive & $4(3.67)$ & $3(10.00)$ & $1(1.27)$ & $0.063^{\mathrm{a}}$ \\
\hline Transfusion & $7(6.42)$ & $2(6.67)$ & $5(6.33)$ & $1.000^{\mathrm{a}}$ \\
\hline
\end{tabular}

COVID-19, coronavirus disease 2019; CHB, chronic hepatitis B; $n$, number.

${ }^{b}$ Treatments include antibiotics (cephalosporin, fluoroquinolones, or macrolides), antiviral therapy (lopinavir/ritonavir, ganciclovir, riboviron, or oseltamivir), or transfusion (suspended red blood cells, platelets, or plasma).

$P$ values were calculated by Fisher's exact test (a) or Pearson's $\chi^{2}$ test. ${ }^{*} P<0.05$. 
Table 4 Factors associated with the illness severity of COVID-19 patients with CHB

\begin{tabular}{|c|c|c|c|c|}
\hline \multirow{2}{*}{ Variables } & \multicolumn{2}{|c|}{ Univariable logistic regression } & \multicolumn{2}{|c|}{ Multivariable logistic regression } \\
\hline & OR $(95 \% \mathrm{CI})$ & $P$ value & OR $(95 \% \mathrm{CI})$ & $P$ value $^{\mathrm{a}}$ \\
\hline \multicolumn{5}{|l|}{ Laboratory examinations } \\
\hline \multicolumn{5}{|l|}{ Blood routine } \\
\hline Neutrophils, \# $(N=107), \times 10^{9} / \mathrm{L}$ & $1.243(1.095-1.412)$ & $0.001 *$ & $1.236(1.078-1.418)$ & $0.002 *$ \\
\hline \multicolumn{5}{|l|}{ Immune cell subsets } \\
\hline Lymphocytes, \# $(N=109), \times 10^{9} / \mathrm{L}$ & $0.274(0.110-0.683)$ & $0.005^{*}$ & $0.274(0.100-0.749)$ & $0.012 *$ \\
\hline $\mathrm{CD}^{+}{ }^{+} \mathrm{CD} 19^{-} \mathrm{T}$ cells, $\#(N=15), / \mu \mathrm{L}$ & $0.995(0.991-0.999)$ & $0.022 *$ & $0.995(0.990-1.000)$ & $0.031 *$ \\
\hline $\mathrm{CD}^{+}{ }^{+} \mathrm{T}$ cells, $\#(N=35), / \mu \mathrm{L}$ & $0.996(0.992-1.000)$ & $0.029 *$ & $0.995(0.991-1.000)$ & $0.038 *$ \\
\hline \multicolumn{5}{|l|}{ Inflammatory cytokines and biomarkers } \\
\hline IL-6 $(N=69), \mathrm{pg} / \mathrm{mL}$ & $1.032(1.005-1.060)$ & $0.021 *$ & $1.032(1.007-1.058)$ & $0.013 *$ \\
\hline $\mathrm{TNF}-\alpha(N=58), \mathrm{pg} / \mathrm{mL}$ & $1.211(1.046-1.402)$ & $0.010^{*}$ & $1.184(1.007-1.393)$ & $0.040 *$ \\
\hline hs-CRP $(N=94), \mathrm{mg} / \mathrm{L}$ & $1.013(1.003-1.023)$ & $0.013 *$ & $1.014(1.002-1.025)$ & $0.018 *$ \\
\hline \multicolumn{5}{|l|}{ Organ damage indexes } \\
\hline $\operatorname{ALT}(N=108), \mathrm{U} / \mathrm{L}$ & $1.011(1.003-1.019)$ & $0.005^{*}$ & $1.012(1.004-1.020)$ & $0.003 *$ \\
\hline $\operatorname{AST}(N=108), \mathrm{U} / \mathrm{L}$ & $1.006(1.000-1.012)$ & $0.040 *$ & $1.007(1.001-1.013)$ & $0.027 *$ \\
\hline $\operatorname{ALP}(N=104), \mathrm{U} / \mathrm{L}$ & $1.008(1.000-1.017)$ & $0.044 *$ & 1.009 (1.001-1.017) & $0.030 *$ \\
\hline TBIL $(N=108), \mu \mathrm{mol} / \mathrm{L}$ & $1.056(1.022-1.092)$ & $0.001 *$ & $1.054(1.018-1.091)$ & $0.003 *$ \\
\hline $\operatorname{ALB}(N=106), \mathrm{g} / \mathrm{L}$ & $0.807(0.732-0.890)$ & $<0.001^{*}$ & $0.803(0.723-0.891)$ & $<0.001^{*}$ \\
\hline $\operatorname{ALB} / \operatorname{GLO}(N=106)$ & $0.073(0.014-0.386)$ & $0.002 *$ & $0.062(0.010-0.385)$ & $0.003 *$ \\
\hline $\mathrm{LDH}(N=105), \mathrm{U} / \mathrm{L}$ & $1.003(1.000-1.006)$ & $0.023 *$ & $1.004(1.001-1.007)$ & $0.021 *$ \\
\hline Amylase $(N=44), \mathrm{U} / \mathrm{L}$ & $0.983(0.964-1.003)$ & 0.089 & $0.968(0.937-1.000)$ & $0.048 *$ \\
\hline hs-cTnI $(N=79), \mathrm{pg} / \mathrm{mL}$ & $1.001(1.000-1.001)$ & $0.029 *$ & $1.001(1.000-1.001)$ & $0.040 *$ \\
\hline $\operatorname{APTT}(N=101), \mathrm{s}$ & $1.098(1.034-1.165)$ & $0.002 *$ & $1.115(1.042-1.192)$ & $0.002 *$ \\
\hline D-dimer $(N=104), \mu \mathrm{g} / \mathrm{mL}$ & $1.097(1.021-1.178)$ & $0.011 *$ & $1.100(1.022-1.185)$ & $0.012 *$ \\
\hline
\end{tabular}

COVID-19, coronavirus disease 2019; CHB, chronic hepatitis B; OR, odds ratio; CI, confidence interval; CD, cluster of differentiation; IL-6, interleukin-6; TNF- $\alpha$, tumor necrosis factor- $\alpha$; hs-CRP, high-sensitivity C-reactive protein; ALT, alanine aminotransferase; AST, aspartate aminotransferase; TBIL, total bilirubin; ALB, albumin; GLO, globulin; LDH, lactate dehydrogenase; ALP, alkaline phosphatase; hs-cTnI, high-sensitivity cardiac troponin I; APTT, activated partial thromboplastin time. \#, counts.

ORs and $95 \%$ CIs were calculated by univariable and multivariable logistic regression models.

${ }^{a}$ Adjusted for age, sex, and comorbidities.

$* P<0.05$.

through induction of $\mathrm{T}$ cell activation and enhancement of immune suppressive cells by the increased production of lactate [17]. Elevated LDH was also reported to be associated with poor clinical outcomes in severe acute respiratory syndrome (SARS) and Middle East respiratory syndrome (MERS) patients [18,19]. ALB can suppress pro-inflammatory NF- $\kappa B$ activation by inhibiting the expression of TNF- $\alpha$ [20]. Furthermore, ALB was used as an effective predictor of disease severity in MERS patients [21]. The abnormal elevation of liver damage indexes was more serious in COVID-19 patients with CHB than those without $\mathrm{CHB}$, especially in severe cases, which might partly be attributed to the direct invasion of SARSCoV-2 to the liver organ or cells on the basis of the observation of viral particles in liver tissues of COVID-19 patients [22]. However, the underlying mechanisms need to be further investigated.

Of note, the coagulation biomarker D-dimer, which was also found to be correlated with adverse outcomes in COVID-19 patients, was abnormally dysregulated in patients with CHB. D-dimer originates from the formation and lysis of cross-linked fibrin and could be a marker of activation of fibrinolysis [23]. Recent studies had reported that hepatic dysfunction in COVID-19 patients is accompanied by coagulative activation and fibrinolytic pathways [24]. Additionally, hyperfibrinolysis resulting from the disturbances of coagulation and hemostasis in patients with acute or chronic liver disease has always been highlighted $[24,25]$. Moreover, the elevation of D-dimer was the prominent presentation of the initial coagulopathy of COVID-19 [26]. Collectively, these findings might partly account for the influence of D-dimer on increased death risk of COVID-19 patients with CHB, and monitoring this indicator might be helpful for the early surveillance of adverse events.

Apart from typical live injury and coagulation dysfunction, the aggravated inflammatory storm appeared to be another feature in COVID-19 patients with CHB, especially in severe cases in our study. We found that the elevation of multiple inflammatory cytokines or infection- 
A

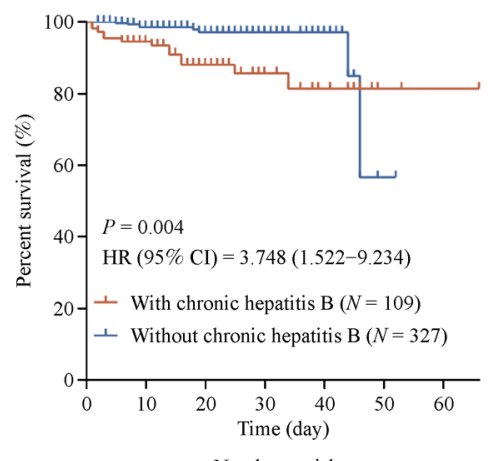

$\begin{array}{llllllll}\text { With CHB } & 109 & 90 & 52 & 24 & 16 & 2 & 2\end{array}$

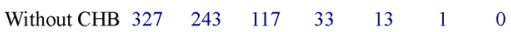

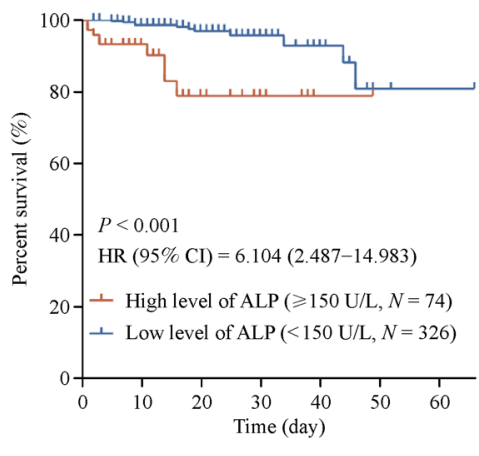

$\begin{array}{llllllll}\text { High ALP } & 74 & 36 & 14 & 6 & 2 & 0 & 0\end{array}$

G

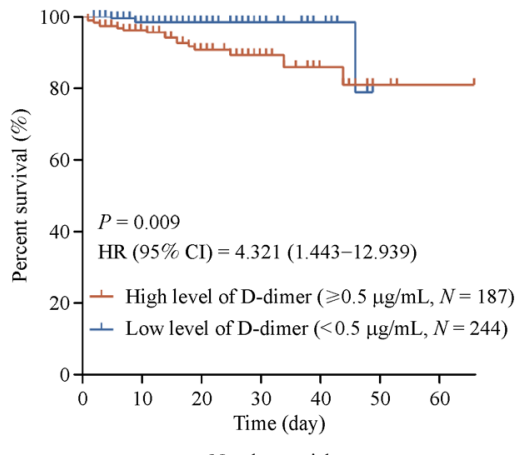

Number at risk

$\begin{array}{llllllll}\text { High D-dimer } & 187 & 154 & 86 & 34 & 18 & 3 & 3\end{array}$

Low D-dimer $\begin{array}{lllllll}244 & 174 & 82 & 23 & 10 & 0 & 0\end{array}$

Fig. 2 Survival of COVID-19 patients during hospitalization. (A) Survival curve of COVID-19 patients with and without CHB. (B-I) Survival curves of COVID-19 patients with different levels of liver test parameters, such as ALT (B), AST (C), ALP (D), TBIL (E), and LDH (F); D-dimer coagulation index $(\mathrm{G})$; ALB $(\mathrm{H})$; and ALB/GLO (I). The survival curves were compared and analyzed, and the hazard ratios (HRs) and 95\% confidence intervals (CIs) were analyzed by the Cox proportional hazards regression models. $P<0.05$ was considered statistically significant.

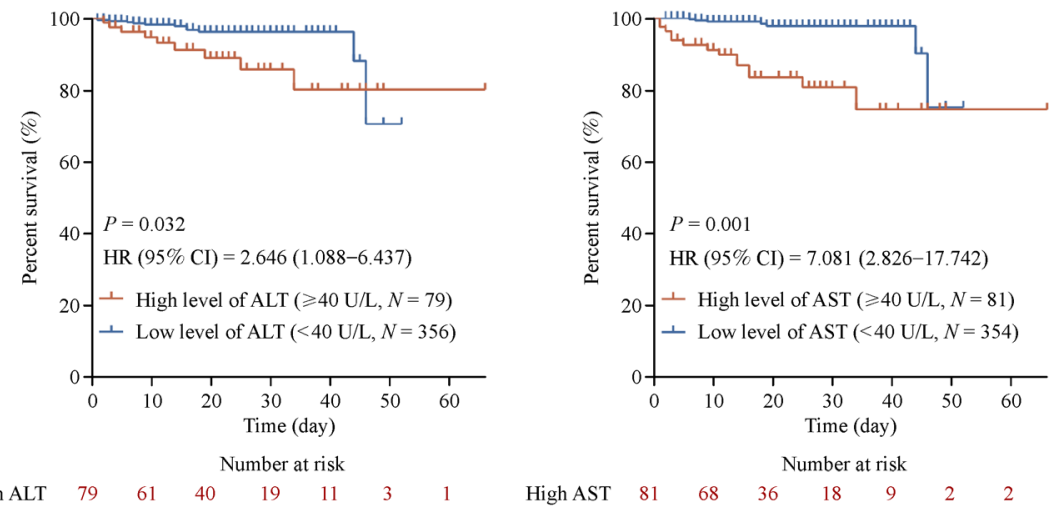

$\mathrm{E}$

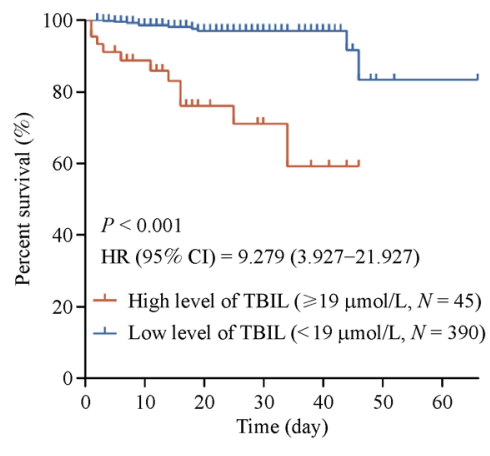

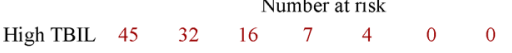

$\mathrm{H}$

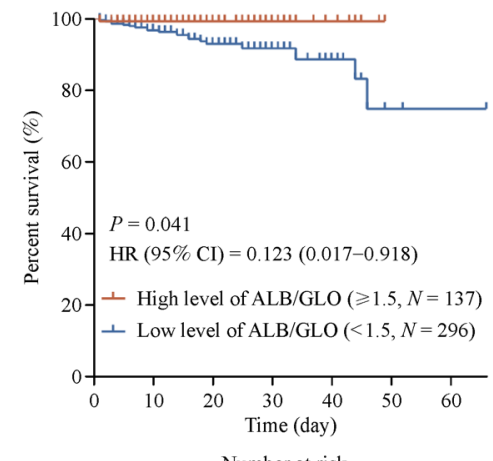

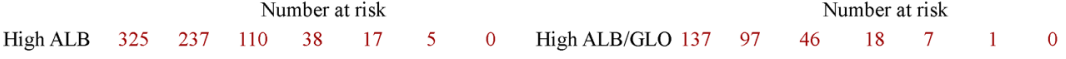

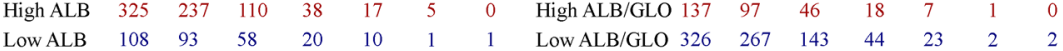


related biomarkers such as TNF- $\alpha$, IL-6, and hs-CRP, as well as neutrophil and leukocyte counts, was more pronounced in COVID-19 patients with CHB than nonCHB patients. The systemic pro-inflammatory cytokine responses after virus infection might induce dysfunction of endothelial cells, resulting in the excess generation of thrombin, which can activate platelets, stimulate fibrinolysis, and predispose to ischemia and thrombosis [26]. Among the measured cytokines, the elevation of IL- 6 was most pronounced. IL- 6 acts as a precipitating factor in the regulation of vascular leakage, complement activation, and coagulation pathway, which partly accounted for poor outcomes for SARS patients with liver injury [27]. However, the association between pro-inflammatory responses and liver injury remains to be explored. Collectively, these findings demonstrated that liver injury, coagulation dysfunction, and aggravated inflammatory responses appeared to be the explicable mechanisms of poor prognosis in COVID-19 patients with CHB, which [27] lead to deleterious complications such as DIC and even death, consistent with recently published studies $[28,29]$.

Effective standardized treatment protocols for COVID19 patients, especially for severe cases, are recommended globally to improve poor outcomes. The combination of antibiotics and antivirals is widely utilized in COVID-19 treatment, and almost half of COVID-19 patients with CHB received antiviral and antibiotic treatment in our study. Consistent with recent studies, which found that severe COVID-19 patients received more clinical interventions $[4,28]$, we found that clinical interventions, especially intravenous immunoglobulin therapy, was more frequently administered in severe cases than in non-severe cases and in $\mathrm{CHB}$ patients than non-CHB patients. Given that no specific treatment has proven to be effective, supportive therapy that could ease the symptoms may be beneficial for decreasing the fatality rate of COVID-19. Considering the uncontrolled inflammatory responses, abnormal coagulation function, and severe liver impairment in COVID-19 patients with CHB, the current management of COVID-19 should be focused on inflammatory reaction and protective treatment for liver function.

Our study also has several limitations. First, this is a retrospective study. Not all laboratory tests were performed in all patients, especially immune cell subtypes. Second, the potential mechanisms of dysregulated inflammatory cytokines for liver injury and the connection between coagulation dysfunction and liver damage need to be further investigated. Third, considering that many examinations were not performed due to the shortage of medical resources during the pandemic, data regarding liver stiffness measurements were not collected in our study. Fourth, given that the prevalence of hepatitis B is much higher than that of other types of hepatitis, our research focused on hepatitis B rather than other types of hepatitis. The impact of other types of hepatitis on COVID-19 remains to be explored. Finally, as COVID-19 patients with CHB are emerging globally, large-scale prospective cohort studies and dynamic measurement of liver injury indicators are warranted.

To our knowledge, this is the largest multi-center retrospective study to date on COVID-19 patients with CHB worldwide, with detailed clinical and laboratory information. In addition to previously reported markers such as ALT, AST, ALP, and TBIL, our study identified several novel risk factors associated with COVID-19 severity and mortality in CHB patients, including elevated LDH and D-dimer and decreased ALB or ALB/GLO.

\section{Acknowledgements}

We thank the assistance of Prof. David Horne from CITY OF HOPE, Prof. Hui Zhu from Cleveland Clinic Foundation, and Prof. Jiping Wang from Northwestern University. This work is supported by the National Natural Science Foundation of China (No. 81974400) and Natural Science Foundation of Zhuhai (No. ZH22036302200081PWC to Renli Deng). We acknowledge all patients and their families involved in the study and all health-care workers who are fighting against the COVID-19 pandemic.

\section{Compliance with ethics guidelines}

Jing Wang, Zequn Lu, Meng Jin, Ying Wang, Kunming Tian, Jun Xiao, Yimin Cai, Yanan Wang, Xu Zhang, Tao Chen, Zhi Yao, Chunguang Yang, Renli Deng, Qiang Zhong, Xiongbo Deng, Xin Chen, Xiang-ping Yang, Gonghong Wei, Zhihua Wang, Jianbo Tian, and Xiao-ping Chen declare that they have no conflict of interest. This study was approved by the Ethics Committee of Tongji Hospital, Tongji Medical College, Huazhong University of Science and Technology and granted a waiver of informed consent from study participants.

Electronic Supplementary Material Supplementary material is available in the online version of this article at https://doi.org/ $10.1007 / \mathrm{s} 11684-021-0854-5$ and is accessible for authorized users.

\section{References}

1. World Health Organization. Rolling updates on coronavirus disease (COVID-19). https://www.who.int/emergencies/diseases/novel-coronavirus-2019 (accessed November 19, 2020)

2. Tian J, Yuan X, Xiao J, Zhong Q, Yang C, Liu B, Cai Y, Lu Z, Wang J, Wang Y, Liu S, Cheng B, Wang J, Zhang M, Wang L, Niu S, Yao Z, Deng X, Zhou F, Wei W, Li Q, Chen X, Chen W, Yang Q, Wu S, Fan J, Shu B, Hu Z, Wang S, Yang XP, Liu W, Miao X, Wang Z. Clinical characteristics and risk factors associated with COVID-19 disease severity in patients with cancer in Wuhan, China: a multicentre, retrospective, cohort study. Lancet Oncol 2020; 21(7): 
893-903

3. Epidemiology Working Group for NCIP Epidemic Response, Chinese Center for Disease Control and Prevention. The epidemiological characteristics of an outbreak of 2019 novel coronavirus diseases (COVID-19) in China. Chin J Epidemiol (Zhonghua Liu Xing Bing Xue Za Zhi) 2020; 41(2): 145-151 (in Chinese)

4. Zhou F, Yu T, Du R, Fan G, Liu Y, Liu Z, Xiang J, Wang Y, Song B, Gu X, Guan L, Wei Y, Li H, Wu X, Xu J, Tu S, Zhang Y, Chen H, Cao B. Clinical course and risk factors for mortality of adult inpatients with COVID-19 in Wuhan, China: a retrospective cohort study. Lancet 2020; 395(10229): 1054-1062

5. GBD 2017 Disease and Injury Incidence and Prevalence Collaborators. Global, regional, and national incidence, prevalence, and years lived with disability for 354 diseases and injuries for 195 countries and territories, 1990-2017: a systematic analysis for the Global Burden of Disease Study 2017. Lancet 2018; 392(10159): 1789-1858

6. Cai Q, Huang D, Yu H, Zhu Z, Xia Z, Su Y, Li Z, Zhou G, Gou J, Qu J, Sun Y, Liu Y, He Q, Chen J, Liu L, Xu L. COVID-19: abnormal liver function tests. J Hepatol 2020; 73(3): 566-574

7. Fix OK, Hameed B, Fontana RJ, Kwok RM, McGuire BM, Mulligan DC, Pratt DS, Russo MW, Schilsky ML, Verna EC, Loomba R, Cohen DE, Bezerra JA, Reddy KR, Chung RT. Clinical best practice advice for hepatology and liver transplant providers during the COVID-19 pandemic: AASLD expert panel consensus statement. Hepatology 2020; 72(1): 287-304

8. Xu Z, Shi L, Wang Y, Zhang J, Huang L, Zhang C, Liu S, Zhao P, Liu H, Zhu L, Tai Y, Bai C, Gao T, Song J, Xia P, Dong J, Zhao J, Wang FS. Pathological findings of COVID-19 associated with acute respiratory distress syndrome. Lancet Respir Med 2020; 8(4): 420 422

9. Veselka J, Faber L, Liebregts M, Cooper R, Januska J, Kashtanov M, Dabrowski M, Hansen PR, Seggewiss H, Hansvenclova E, Bundgaard H, Ten Berg J, Stables RH, Jensen MK. Short- and longterm outcomes of alcohol septal ablation for hypertrophic obstructive cardiomyopathy in patients with mild left ventricular hypertrophy: a propensity score matching analysis. Eur Heart J 2019; 40(21): 1681-1687

10. Sarin SK, Kumar M, Lau GK, Abbas Z, Chan HL, Chen CJ, Chen DS, Chen HL, Chen PJ, Chien RN, Dokmeci AK, Gane E, Hou JL, Jafri W, Jia J, Kim JH, Lai CL, Lee HC, Lim SG, Liu CJ, Locarnini S, Al Mahtab M, Mohamed R, Omata M, Park J, Piratvisuth T, Sharma BC, Sollano J, Wang FS, Wei L, Yuen MF, Zheng SS, Kao JH. Asian-Pacific clinical practice guidelines on the management of hepatitis B: a 2015 update. Hepatol Int 2016; 10(1): 1-98

11. World Health Organization. Clinical management of severe acute respiratory infection when Novel coronavirus $(\mathrm{nCoV})$ infection is suspected: interim guidance. 2020. https://www.who.int/publications-detail/clinical-management-of-severe-acute-respiratory-infection-when-novel-coronavirus-(ncov)-infection-is-suspected (accessed May 23, 2020)

12. National Health Commission of the People's Republic of China. Chinese management guideline for COVID-19 (version 7.0, in Chinese). Updated: March 3, 2020. 2020. http://www.nhc.gov.cn/ yzygj/s7653p/202003/46c9294a7dfe4cef80dc7f5912eb1989/files/ ce3e6945832a438eaae415350a8ce964.pdf (accessed May 23, 2020)

13. Levi M, Toh $\mathrm{CH}$, Thachil J, Watson HG. Guidelines for the diagnosis and management of disseminated intravascular coagulation. British Committee for Standards in Haematology. Br J Haematol 2009; 145(1): 24-33

14. Chen G, Wu D, Guo W, Cao Y, Huang D, Wang H, Wang T, Zhang X, Chen H, Yu H, Zhang X, Zhang M, Wu S, Song J, Chen T, Han M, Li S, Luo X, Zhao J, Ning Q. Clinical and immunological features of severe and moderate coronavirus disease 2019. J Clin Invest 2020; 130(5): 2620-2629

15. Bai Y, Yao L, Wei T, Tian F, Jin DY, Chen L, Wang M. Presumed asymptomatic carrier transmission of COVID-19. JAMA 2020; 323(14): 1406-1407

16. Lei F, Liu YM, Zhou F, Qin JJ, Zhang P, Zhu L, Zhang XJ, Cai J, Lin L, Ouyang S, Wang X, Yang C, Cheng X, Liu W, Li H, Xie J, Wu B, Luo H, Xiao F, Chen J, Tao L, Cheng G, She ZG, Zhou J, Wang H, Lin J, Luo P, Fu S, Zhou J, Ye P, Xiao B, Mao W, Liu L, Yan Y, Liu L, Chen G, Li H, Huang X, Zhang BH, Yuan Y. Longitudinal association between markers of liver injury and mortality in COVID-19 in China. Hepatology 2020; 72(2): 389-398

17. Ding J, Karp JE, Emadi A. Elevated lactate dehydrogenase (LDH) can be a marker of immune suppression in cancer: interplay between hematologic and solid neoplastic clones and their microenvironments. Cancer Biomark 2017; 19(4): 353-363

18. Al Ghamdi M, Alghamdi KM, Ghandoora Y, Alzahrani A, Salah F, Alsulami A, Bawayan MF, Vaidya D, Perl TM, Sood G. Treatment outcomes for patients with Middle Eastern respiratory syndrome coronavirus (MERS CoV) infection at a coronavirus referral center in the Kingdom of Saudi Arabia. BMC Infect Dis 2016; 16(1): 174

19. Tsui PT, Kwok ML, Yuen H, Lai ST. Severe acute respiratory syndrome: clinical outcome and prognostic correlates. Emerg Infect Dis 2003; 9(9): 1064-1069

20. Spinella R, Sawhney R, Jalan R. Albumin in chronic liver disease: structure, functions and therapeutic implications. Hepatol Int 2016; 10(1): 124-132

21. Saad M, Omrani AS, Baig K, Bahloul A, Elzein F, Matin MA, Selim MA, Al Mutairi M, Al Nakhli D, Al Aidaroos AY, Al Sherbeeni N, Al-Khashan HI, Memish ZA, Albarrak AM. Clinical aspects and outcomes of 70 patients with Middle East respiratory syndrome coronavirus infection: a single-center experience in Saudi Arabia. Int J Infect Dis 2014; 29: 301-306

22. Wang Y, Liu S, Liu H, Li W, Lin F, Jiang L, Li X, Xu P, Zhang L, Zhao L, Cao Y, Kang J, Yang J, Li L, Liu X, Li Y, Nie R, Mu J, Lu F, Zhao S, Lu J, Zhao J. SARS-CoV-2 infection of the liver directly contributes to hepatic impairment in patients with COVID-19. J Hepatol 2020; 73(4): 807-816

23. Zhang L, Yan X, Fan Q, Liu H, Liu X, Liu Z, Zhang Z. D-dimer levels on admission to predict in-hospital mortality in patients with Covid-19. J Thromb Haemost 2020; 18(6): 1324-1329

24. Qi T, Zhu C, Lu G, Hao J, He Q, Chen Y, Zhou F, Chen J, Hou J. Elevated D-dimer is associated with increased 28-day mortality in acute-on-chronic liver failure in China: a retrospective study. BMC Gastroenterol 2019; 19(1): 20

25. Tripodi A. Hemostasis in acute and chronic liver disease. Semin Liver Dis 2017; 37(1): 28-32

26. Connors JM, Levy JH. COVID-19 and its implications for thrombosis and anticoagulation. Blood 2020; 135(23): 2033-2040

27. Duan ZP, Chen Y, Zhang J, Zhao J, Lang ZW, Meng FK, Bao XL. 
Clinical characteristics and mechanism of liver injury in patients with severe acute respiratory syndrome. Chin J Hepatol (Zhonghua Gan Zang Bing Za Zhi) 2003; 11(8): 493-496 (in Chinese)

28. Cao X. COVID-19: immunopathology and its implications for therapy. Nat Rev Immunol 2020; 20(5): 269-270
29. Chen T, Wu D, Chen H, Yan W, Yang D, Chen G, Ma K, Xu D, Yu H, Wang H, Wang T, Guo W, Chen J, Ding C, Zhang X, Huang J, Han M, Li S, Luo X, Zhao J, Ning Q. Clinical characteristics of 113 deceased patients with coronavirus disease 2019: retrospective study. BMJ 2020; 368: m1091 